\title{
Micro-Raman study of the Allende meteoritic nanodiamonds: Supernova-driven shock wave origin is revisited
}

\author{
Arnold Gucsik \\ Konkoly Observatory, Hungarian Academy of Sciences, Budapest
}

\begin{abstract}
I have studied the Raman spectroscopic signatures of nanodiamonds from the Allende meteorite, in which some portions must be of presolar origin as indicated by the isotopic compositions of various trace elements. The spectra of the meteoritic nanodiamonds show a narrow peak at $1326 \mathrm{~cm}^{-1}$ and a broad band at $1590 \mathrm{~cm}^{-1}$. Compared to the intensities of these peaks, the background fluorescence is relatively high. A significant frequency shift from 1332 to $1326 \mathrm{~cm}^{-1}$, peak broadening, and appearance of a new peak at $1590 \mathrm{~cm}^{-1}$ might be due to shock effects during formation of the diamond grains. Such changes may have several origins: an increase in bond length, a change in the electron density function or charge transfer, or a combination of these factors. However, Raman spectroscopy alone does not allow distinguishing between a shock origin of the nanodiamonds and formation by a CVD process, as is favored by most workers.
\end{abstract}

Key words: presolar grains, nanodiamond, shock waves, Raman spectroscopy

\section{Introduction}

Primitive meteorites contain abundant (up to $1500 \mathrm{ppm}$ ) amounts of nanodiamonds. At least some subpopulation must be of pre-solar (stardust?) origin, as indicated by the isotopic composition of trace elements the diamonds carry, in particular noble gases (Huss and Lewis 1994) and tellurium (Richter et al. 1998). On the other hand, the isotopic composition of the major element, carbon, is unremarkable, i.e. it is within the range reasonably expected for Solar System materials (Russel et al. 1992). As a consequence many workers believe that

\footnotetext{
Address: A. Gucsik: H-1121 Budapest, Konkoly-Thege út 15-17, Hungary, e-mail: ciklamensopron@yahoo.com

Received: November 11, 2011; accepted November 30, 2011
} 
the majority of the diamonds is of local, i.e. Solar System, origin and that the fraction that is pre-solar is relatively small (Zinner 1998). Two main theories exist for the formation process of the meteoritic nanodiamonds (e.g. Daulton et al. 1996 - and references therein): (1) Chemical vapor deposition (CVD), and (2) shock origin. TEM investigations, in particular, seem to suggest that formation by a CVD process is most likely (Daulton et al. 1996).

In this study, I present results of the study of meteoritic nanodiamonds from the Allende meteorite by means of Raman spectroscopy in an attempt to obtain further constraints with regard to the formation process and further interpretation of data obtained by Gucsik et al. (2008a).

\section{Materials and methods}

The Allende meteorite is a carbonaceous chondrite of type CV3 (Fig. 1). It is the meteorite where nanodiamonds, in the search for the carrier of isotopically anomalous noble gases, were first identified (Lewis et al. 1987). The abundance of nanodiamonds in this meteorite is $\sim 250 \mathrm{ppm}$ (Huss and Lewis 1995). The nanodiamonds investigated in this work were extracted from the meteorite using a variant of the microwave digestion procedure.

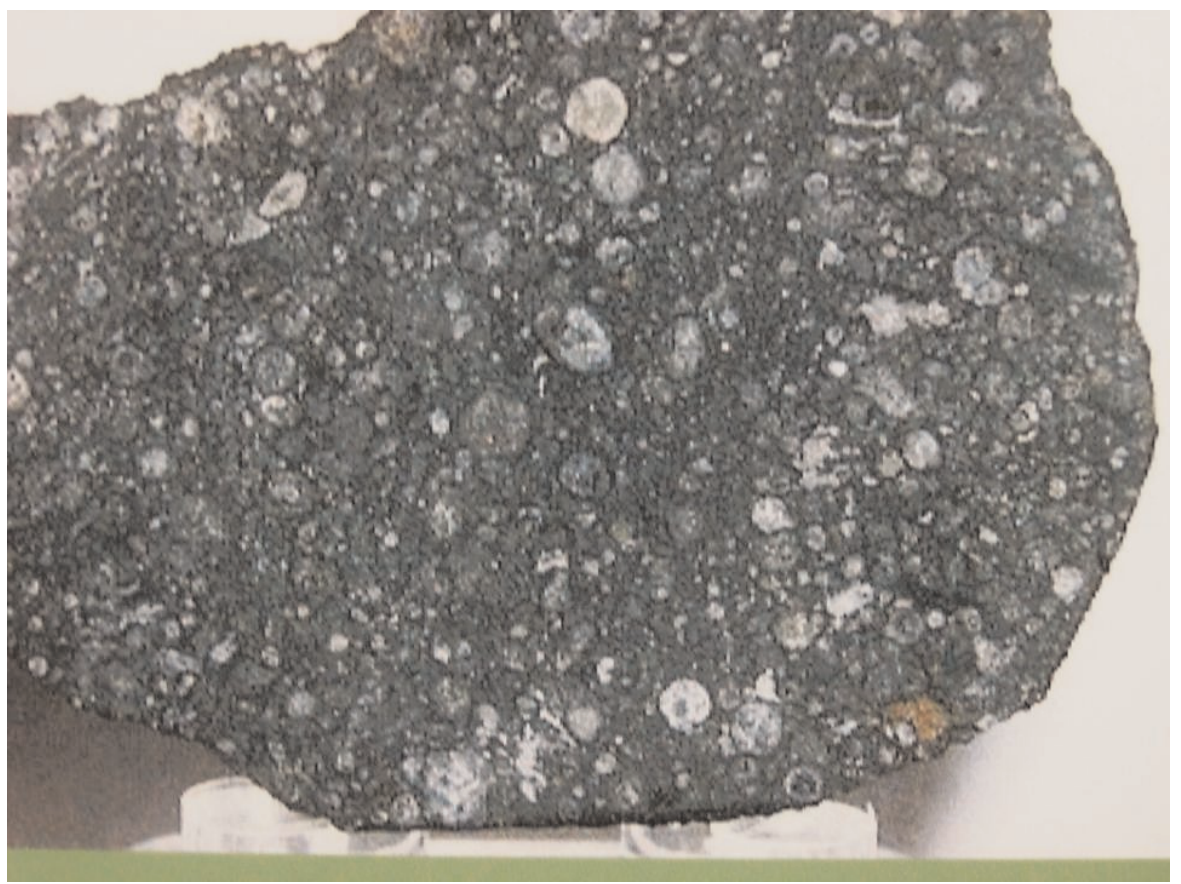

Fig. 1

A piece of the Allende meteorite showing typical features of the carbonaceous chondrite such as chondrules (white parts) (photo by author) 
Raman spectra were recorded using the confocal Raman micro-spectrometer T64000 (Jobin-Yvon) equipped with a BX-40 (Olympus) microscope at Technical University of Lodz, Poland. A small amount (less than 1 cubic micrometer) of nanodiamonds was placed on a microscope slide using an acupuncture needle and covered with a thin microcover glass. The slide was carefully positioned under the objective $(\times 50)$, which was used to focus the excitation laser light (Argon line $=514.5 \mathrm{~nm}$ ) onto the sample. The output power of the laser was $200 \mathrm{~mW}$ ( $\sim 2 \mathrm{~mW}$ on the sample). A high background, which is typical for nanomaterials, limited the acquisition time to 90-300 s, dependent on the specific sample. In order to increase the signal/noise ratio two accumulation cycles were used. All spectra contain a significant contribution from the glass (peaks at 580, 790,1100 , and $1900 \mathrm{~cm}^{-1}$ ) as a background, because the laser spot was larger than the diameter of the sample.

\section{Results and discussions}

Raman spectral features of the meteoritic nanodiamonds

The individual grain sizes of meteoritic nanodiamonds vary between 2 and 7 $\mathrm{nm}$ (e.g. Daulton et al. 1996), which is small compared to the $\sim 1 \mu \mathrm{m}$ diameter of the laser excitation beam on the surface of the sample. The Raman spectra of the nanodiamonds exhibit two broad bands centered at $\sim 1326$, and $\sim 1590 \mathrm{~cm}^{-1}$. In general, peak intensities of these bands are relatively low, which indicates strong background fluorescence. This may be due to lack of well-formed crystalline parts of the sample; alternatively, it may (also) be related to the small grain size. Following data correction, the band at $1326 \mathrm{~cm}^{-1}$ shows $14.4 \mathrm{~cm}^{-1} \mathrm{FWHM}$ and a $6 \mathrm{~cm}^{-1}$ peak shift from the $1332 \mathrm{~cm}^{-1}$ peak position of standard or reference nanodiamond (Ferrari and Robertson 2001, 2004; Zhang and Zhang 2005; Karczemska 2010) (Fig. 2).

The Raman spectra of single crystal diamond is dominated by a Brillouin zonecenter point as T2g mode at $1332 \mathrm{~cm}^{-1}$ (strong or very strong peak for carbon sp3 bonding) with approximately 5-10 $\mathrm{cm}^{-1}$ FWHM (Ferrari and Robertson 2001, 2004; Zhang and Zhang 2005). This relatively sharp and single peak is frequently used as a signature of high crystalline quality. In previous studies of micro- and nanodiamond samples several additional peaks have been described in the Raman spectra, as follows. The two most typical and significant ones in the spectra of artificially-produced chemical vapor deposited (CVD) nanocrystalline diamonds are at 1150 and $1450 \mathrm{~cm}^{-1}$ (trans-polyacetylenes; Ferrari and Robertson 2001; Karczemska 2010). It has been proposed that these peaks are related to phonon modes with q_0, which are activated by the disorder induced by small grain sizes in nanocrystalline or amorphous diamond (Filik et al. 2006). Additional medium or strong bands in the Raman spectra of nanocrystalline diamond samples are usually seen at 1350 and at $1590 \mathrm{~cm}^{-1}$. The $1350 \mathrm{~cm}^{-1}$ feature is related to the D-band, which is a normally Raman inactive A1g mode. 


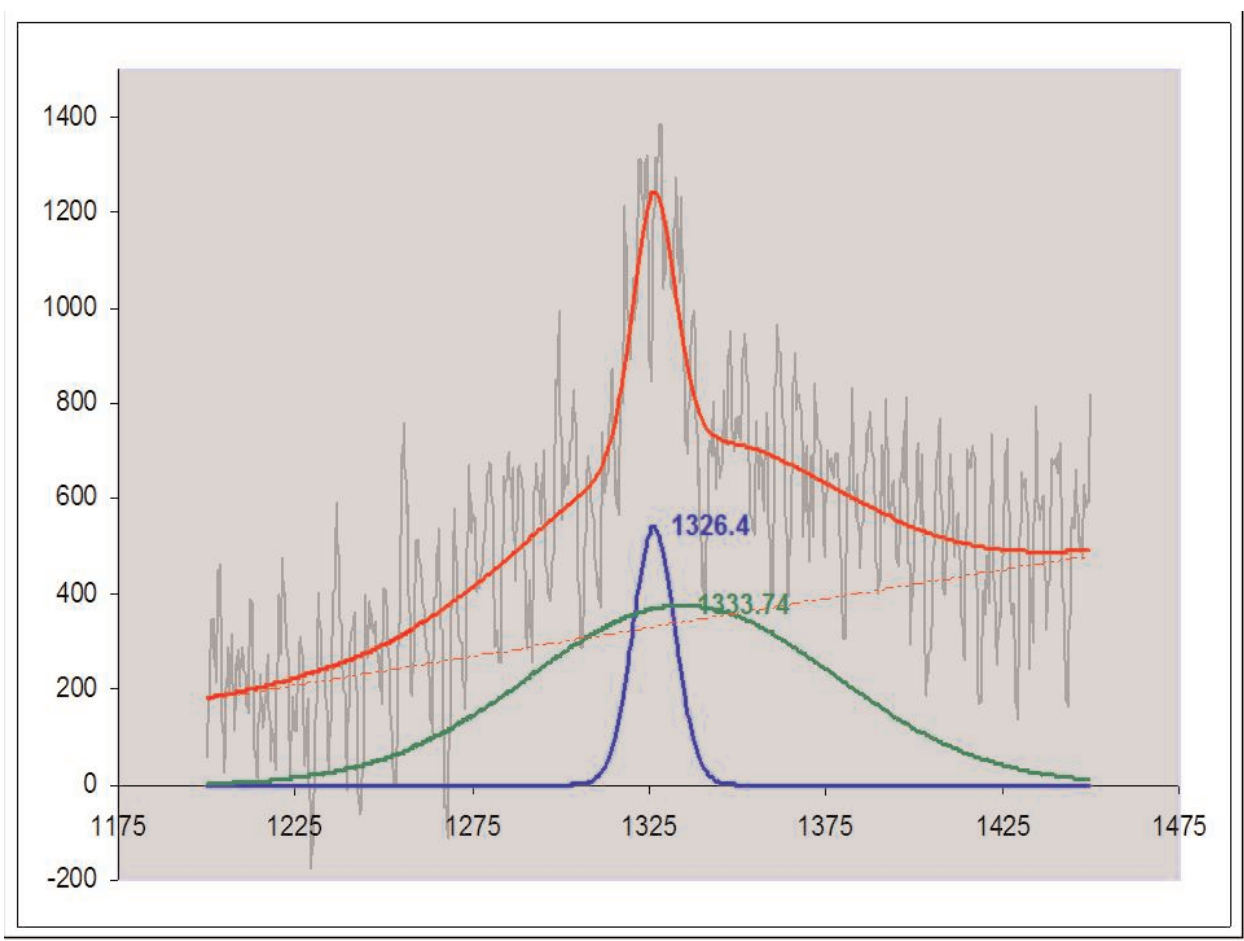

Fig. 2

Micro-Raman spectral features of the Allende meteoritic nanodiamonds. Data correction of a peak at $1326 \mathrm{~cm}^{-1}$

It is activated due to the finite crystal size (Filik et al. 2006 - and references therein). The G-band at $1590 \mathrm{~cm}^{-1}$ is assigned to carbon sp2 bonding graphitic structures (Nasdala et al. 2004).

\section{Raman frequency shift}

Frequency shifts of the $1332 \mathrm{~cm}^{-1}$ band by a few cm $\mathrm{cm}^{-1}\left(6 \mathrm{~cm}^{-1}\right.$ in this case) may be due to strained nanodiamond caused by shock waves or high-pressureinduced deformation but also due to disorder in the nanodiamond structure. Similar frequency shifts, broad bands $\left(10-20 \mathrm{~cm}^{-1}\right.$ at FWHM) and relatively high background fluorescence were observed in impact-induced diamond as well as in nanodiamond samples from different shock metamorphic environments such as terrestrial impact structures (El Goresy et al. 2001) and meteorites (Greshake et al. 2000; Mostefaoui et al. 2002; Gucsik et al. 2008a and b; Karczemska et al. 2009) as well as in shock recovery experiments (Kenkmann et al. 2002). We note, however, that the frequency shift of the band at $1332 \mathrm{~cm}^{-1}$ and the peak broadening from higher modes to the lower ones may also be assigned to the 
effects of phonon/quantum confinement (Yoshikawa et al. 1995; Chen et al. 1999; Sun et al. 2000; Berg et al. 2008). Shifts were also observed after irradiation by neutrons (Guo et al. 2004). An additional peak at $1590 \mathrm{~cm}^{-1}$ was also described in the previous studies, which is probably related to the amorphous carbon phase present as the result of shock metamorphism (El Goresy et al. 2001; Kenkmann et al. 2002). These observations on shock-produced diamonds are in a good agreement with the Raman spectral properties of nanodiamonds from our Allende meteorite sample. The frequency shift may be associated with the modification of the local configuration of the sample by means of the transformation of graphite into nanodiamond (Dunlop et al. 2007). In particular, the difference in frequency values for the Raman C-C bonding vibrations between 1332 and $1326 \mathrm{~cm}^{-1}$ in nanodiamond could indicate a change of the C$\mathrm{C}$ bond strength caused by the phase transition at high pressure and temperature.

\section{Shock wave scenario}

It is well known (based on shock recovery experiments) that the required shock pressure to produce impact-induced diamonds is around $40 \mathrm{GPa}$ (Kenkmann et al. 2002). Since the Allende meteorite does not contain high-pressure polymorphs as evidence for a high shock stage, impact on the Allende parent body cannot be responsible for the formation of the nanodiamonds. This, of course, is also evident from the fact that nanodiamonds are present not just in Allende but in all primitive meteorites, with their relative abundance largely determined by losses due to metamorphism (Huss 1990; Huss and Lewis 1995). According to Klein et al. (1994) interstellar shock waves can be produced by the powerful stellar winds of massive stars, supernova explosions, cloud-cloud collisions, and spiral density waves. In the following paragraph I focus on the interaction of supernova-driven shock waves and interstellar cloud and their relationship to the linear growth rate of nanodiamonds.

Assuming a supernova shock wave front is responsible for the synthesis of diamond, its formation can be considered as a two-step process. According to the supernova shock wave experiment of Hansen et al. (2007) the first step is the transition of the secondary shock wave through interstellar media near the supernova explosion, resulting in grain-grain collisions. The initial shock wave occurs rapidly, which can provide the physical conditions such as high pressure and high temperature adequate for the formation of diamond, creating the second stage or the crystallization stage. A disadvantage of this process, however, is that pressures obtained during such collisions are often so high that shattering, sputtering and vaporization become dominant over phase transformation (Tielens et al. 1987; Jones et al. 1994, 1996), which limits the effectiveness of diamond formation. In this stage, according to the linear growth rate of diamond 
(RD), the following relation between the linear size $\mathrm{L}$ of the growing diamond, the linear growth rate RD and growth time (time of crystallization) is given by

$$
L(n m)=R D(n m / s) t(s)
$$

For CVD diamonds, the RD rate of CVD ranges between 0.03 and $1.2-1.7 \mathrm{~nm} / \mathrm{s}$. Accordingly, for the meteoritic nanodiamonds with a mean size L of 2.6 nanometers the time of crystallization ( $t$ ) would be between 1-2 s for relatively rapid growth and on the order of $100 \mathrm{~s}$ for slow growth. The structural property (i.e. startwin structure) of meteoritic nanodiamonds favors rapid growth and several seconds. In the case of the high pressure/high temperature (HPHT) processes, growth rates are high, in the order of $300-500 \mathrm{~nm} / \mathrm{s}$. This would result in growth times of $0.005-0.01$ s (i.e. 5-10 ms), much shorter than for CVD processes (Battaile et al. 1997; Chung and Sung 2001; Aleksandrov and Sel'skaya 2002; Abbashian et al. 2005; Mokuno et al. 2006; Karczemska et al. 2008). According to Boss et al. (2008) the speed of the shock wave was calculated in a simulation of the triggered collapse of a presolar dense cloud core. In that case the transition would be $2.6 \mathrm{~nm} / 20 \mathrm{~km} / \mathrm{sec}$ (=0.13 picoseconds). It is also poorly understood whether or not nanodiamonds would be formed during such a very short period in the Universe. Of course, more detailed experimental work must be carried out focusing on the relationship to relatively rapid growth rate and speed of the shock wave front (Fig. 3).

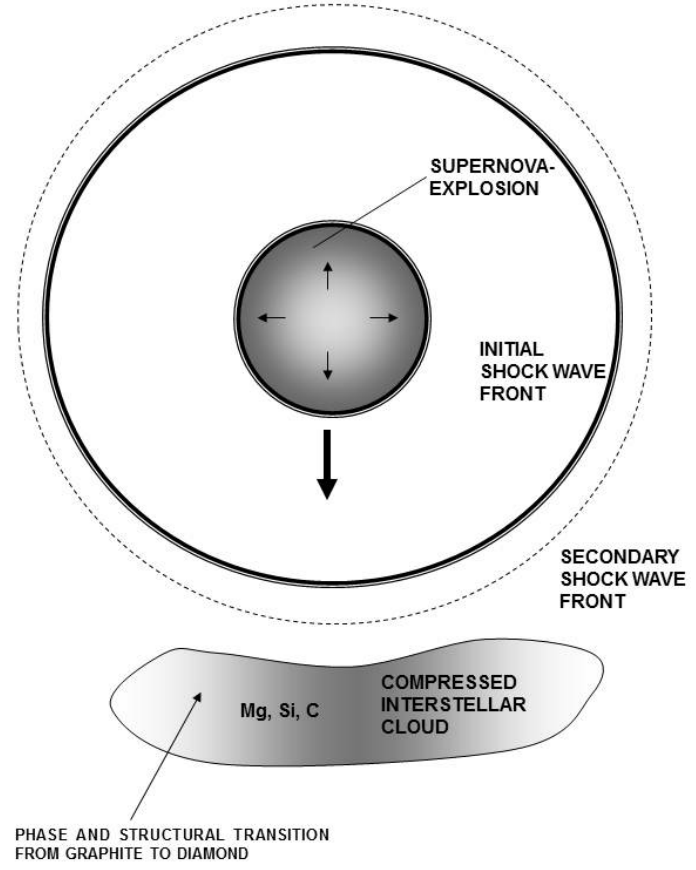

Fig. 3

A schematic figure showing a possible way to nanodiamond formation in the compressed interstellar cloud due to double supernova shock wave fronts following the Hansen model (Hansen et al. 2007). Scale: a few hundred light years in diameter 
Alternatively, it is also important to note that the $2.6 \mathrm{~nm}$ average size of the meteoritic nanodiamonds may be related to the $\mathrm{H}$-related self-terminating crystal growth process (Sun et al. 2004) in the shock-wave front. On the other hand, the surface chemistry of the nanodiamonds shows a poor thermal stability between $850-1350{ }^{\circ} \mathrm{C}$ (Lu et al. 2007), indicating the low-temperature crystallization process of the single diamond grains. These factors may also support the shock wave origin of the meteoritic nanodiamonds.

\section{Conclusions}

These results from Raman spectroscopy alone are not conclusive, especially since it is currently difficult to distinguish between the effects of shock transformation and small grain size. They leave open, however, the possibility that a significant fraction of the nanodiamonds in primitive meteorites were formed by shock transformation from graphite/amorphous carbon in the interstellar medium. As noted above, this possibility was immediately recognized following their discovery (Tielens et al. 1987), but more recent work has mostly concluded that a CVD-like process is more likely (e.g. Daulton et al. 1996; Le Guillou et al. 2006, 2007). Other processes that are possible in principle (see also Anders and Zinner 1998), but have received less attention, are photolysis of hydrocarbons (Buerki and Leutwyler 1991), annealing by UV photons (Nuth and Allen 1992), and transformation by energetic particle irradiation (e.g. Ozima and Tatsumoto 1997). Note that Raman peak shifts were observed after irradiation by neutrons (Chen et al. 2004), but otherwise information concerning the effects of these processes on the Raman spectral properties of nanodiamonds is lacking. As further measurements, it is also planned to use a multiple technological approach such as combination of Raman spectroscopy with Scanning Electron MicroscopeCathodoluminescence (SEM-CL) spectroscopy (Gucsik et al. 2009) and microscopy to carry out detailed analyses on the meteoritic nanodiamonds.

\section{Aknowledgements}

The author would like to express his thanks to Drs Karczemska, Kozanecki, Mitura, and Szurgot at the University of Lodz (Poland), and to Dr Ulrich Ott (Max Planck Institute for Chemistry, Mainz, Germany) for the Micro-Raman spectroscopic data and useful comments on this manuscript, as well as to Prof. Bérczi (Eötvös Loránd University, Budapest) for his review of this paper. The author is grateful for the Cambridge University Press for the publication permission given for the Gucsik et al. (2008) Micro-Raman study of nanodiamonds from Allende meteorite. Proceedings of the International Astronomical Union, Organic Matter in Space Congress-251, Volume 4, pp. 335-340, wich was further discussed in this study, as well. 


\section{References}

Abbashian, R., H. Zhu, F. Clarke 2005: High pressure-high temperature growth of diamond crystals using split sphere apparatus. - Diamond and Related Materials, 14, pp. 1916-1919.

Aleksandrov, V.D., I.V. Sel'skaya 2002: Effect of synthesis conditions on the growth rate and structure of diamond films. - Inorganic Materials, 39, pp. 455-458.

Anders, A., E. Zinner 1998: Interstellar grains in primitive meteorites - Diamond, silicon carbide, and graphite. - Meteoritics, 28, pp. 490-514.

Battaile, C.C., D.J. Srolovitz, J.E. Butler 1997: Molecular view of diamond CVD growth. - Journal of Electronic Materials, 26, pp. 960-965.

Berg, T., E. Marosits, J. Maul, P. Nagel, U. Ott, F. Schertz, S. Schuppler, C. Sudek, G. Schönhense 2008: Quantum confinement observed in the X-ray absorption spectrum of size distributed meteoritic nanodiamonds. - Applied Physics Letters, 104, 064303.

Buerki, P.R., S. Leutwyler 1991: Homogeneous nucleation of diamond powder by CO2-laser-driven gas-phase reactions. - Journal Applied Physics, 69, pp. 37-39.

Boss, A.P., S.I. Ipatov, S.A. Keiser, E.A.M. Harri, T.A. Vanhala 2008: The Astrophysical Journal Letters, 686, L119.

Chen, P., F. Huang, S. Yun 2006: Optical characterization of nanocarbon phases in detonation soot and shocked graphite. - Diamond Related Materials, 15, pp. 1400-1404.

Chen, P., F. Huang, S. Yun 2004: Structural analysis of dynamically synthesized diamonds. - Material Research Bulletin, 39, pp. 1589-1597.

Chen, J., S.Z. Deng, J. Chen, Z.X. Yu, N.S. Xu 1999: Graphitization of nanodiamond powder annealed in argon ambient. - Applied Physics Letters, 74, pp. 3651-3654.

Chung, H-K., J.C. Sung 2001: The CVD growth of micro crystals of diamond. - Diamond and Related Materials, 10, pp. 1584-1587.

Daulton, T.L., D.D. Eisenhour, T.J. Bernatowitz, R.S. Lewis, P.R. Buseck 1996: Genesis of presolar diamonds: Comparative high-resolution transmission electron microscopy study of meteoritic and terrestrial nano-diamonds. - Geochimica et Cosmochimica Acta, 60, pp. 4853-4872.

Dunlop, A., G. Jaskierowitz, P.M. Ossi, S. Della-Negra 2007: Transformation of graphite into nanodiamond following extreme electronic excitations. - Physical Review B, 76, 155403.

Ferrari, A.C., J. Robertson 2004: Raman spectroscopy of amorphous, nanostructured, diamond-like carbon, and nanodiamond. - Philosophical Transactions Royal Society London A, 362, pp. 2477-2512.

Ferrari, A.C., J. Robertson 2001: Origin of the $1150-\mathrm{cm}^{-1}$ Raman mode in nanocrystalline diamond. Physical Review, B. 63, 121405-9.

Filik, J., N. Harvey, N.L. Allan, P.W. May, J.E.P. Dahl, S. Liu, R.M.K. Carlson 2006: Raman spectroscopy of nanocrystalline diamond: An ab initio approach. - Physical Review B, 74, 035423.

El Goresy, A., P. Gillet, M. Chen, F. Künstler, G. Graup, V. Stähle 2001: In situ discovery of shockinduced graphite-diamond phase transition in gneisses from the Ries Crater, Germany. American Mineralogist, 86, pp. 611-621.

Greshake, A., T. Kenkmann, R.T. Scmitt. 2000: In situ Raman spectroscopy of diamond in the ureilite Hammadah Al Hamra 126. - 63rd Meteoritical Society Meeting, abs. \#5049.

Gucsik, A., I. Simonia, K. Ninagawa, H. Nishido, M. Nakazato 2009: Cathododoluminescence study of meteoritic pre-solar nanodiamonds: An implication for origin of diamond particles in NGC 7027 planetary nebula. - Pacific Astronomical Union, Division of Planetary Sciences, 41st Annual Meeting.

Gucsik, A., U. Ott, E. Marosits, A. Karczemska, M. Kozanecki, M. Szurgot 2008a: Micro-Raman study of nanodiamonds from the Allende meteorite. - Proceedings for IAU Symposium 251: Organic Matter in Space. Cambridge University Press 251, pp. 335-338. 
Gucsik, A., A.B. Verchovsky, U. Ott, E. Marosits, A. Karczemska, M. Kozanecki, M. Szurgot 2008b: Meteoritic nanodiamond: a micro-Raman spectroscopical overview. - 39th Lunar and Planetary Science Conference, Houston, U.S.A., abs. \#1201.

Guo, Y., Z. Zheng, Y. Feng, Y. Li 2004: Effects of neutron irradiation on nanodiamond: Raman spectroscopy study. - Journal of Peking University (Science Edition), 40, 212 p.

Le Guillou, C., J.N. Rouzaud 2007: Nanodiamonds graphitization under temperature: Implications on their evolution during chondrites parent body metamorphism. - Lunar and Planetary Science Conference XXXVIII, abs. \#1578

Le Guillou, C., J.N. Rouzaud, F. Brunet 2006: Characterization of the carbon to diamond transition by X-Ray Diffraction, Raman Microspectroscopy, and High Resolution Transmission Electron Microscopy (HRTEM) A way to better constrain the formation of diamond in Space. - Lunar and Planetary Science Conference XXXVIII, abs. \#1635

Hansen, J.F., M.F. Robey, R.I. Klein, A.R. Miles 2007: Experiment on the mass stripping of an interstellar cloud following shock passage. - The Astrophysical Journal, 662, pp. 379-388.

Huss, G.R. 1990: Ubiquitous interstellar diamond and $\mathrm{SiC}$ in primitive chondrites: abundances reflect metamorphism. - Nature, 347, pp. 159-162.

Huss, G.R., R.S. Lewis 1995: Presolar diamond, $\mathrm{SiC}$, and graphite in primitive chondrites: Abundances as a function of meteorite class and petrologic type. - Geochimica et Cosmochimica Acta, 59, pp. 115-160.

Huss, G.R., R.S. Lewis 1994: Noble gases in presolar diamonds I: Three distinct components and their diamond origins. - Meteoritics, 29, pp. 791-810.

Jones, A.P., A.G.G.M. Tielens, D.J. Hollenbach 1996: Grain shattering in shocks: The interstellar grain size distribution. - The Astrophysical Journal, 469, p. 740.

Jones, A.P., A.G.G.M. Tielens, D.J. Hollenbach, C.F. McKee 1994: Grain destruction in shocks in the interstellar medium. - The Astrophysical Journal, 433, pp. 797-810.

Karczemska, T.A. 2010: Diamonds in meteorites-Raman mapping and cathodoluminescence studies. - Journal of Achievements in Materials and Manufacturing Engineering, 43, pp. 94-107.

Karczemska, A., T. Jakubowski, M. Kozanecki, I. Tszydel, A. Jauss, A. Gucsik 2009: Micro-Raman Spectroscopy of Diamonds from JaH 054 and Sahara 98505 Ureilites, Statistic Research. - AIP Proceedings of the International Conference, 1163, pp. 59-74.

Karczemska, A., M. Szurgot, M. Kozanecki, M.I. Szynkowska, V. Ralchenko, V.V. Danilenko, P. Louda, S. Mitura 2008: Extraterrestrial, terrestrial and laboratory diamonds - differences and similarities. - Diamond and Related Materials, 17, pp. 1179-1185.

Kenkmann, T, U. Hornemann, D. Stöfler 2002: Transformation of graphite to diamond in shock experiments: A Raman study. - Lunar and Planetary Science Conference XXXIII, abs. \#1052.

Klein, R.I., Ch.F. McKee, Ph. Colella 1994: On the hydrodynamic interaction of shock waves with interstellar clouds. 1: Nonradiative shocks in small clouds. - The Astrophysical Journal, 420, pp. 213-236.

Lewis, R.S., T. Ming, J.F. Wacker, E. Andres, E. Steel 1987: Interstellar diamonds in meteorites. Nature, 326, pp. 160-162.

Lu, J., Y.H. Wang, J.B. Zang, Y.N. Li 2007: Protective silicone coating for nanodiamonds using atomic layer deposition. - Applied Surface Science, 253, pp. 3485-3488.

Mostefaoui, S., A. El Goresy, P. Hoppe, Ph. Gillet, U. Ott 2002: Mode of occurrence, textural settings and nitrogen-isotopic compositions of in situ diamonds and other carbon phases in the Bencubbin meteorite. - Earth and Planetary Science Letters, 204, pp. 89-100.

Mokuno, Y., A. Chayahara, Y. Soda, H. Yamada, Y. Horino, N. Fujimori 2006: High rate homoepitaxial growth of diamond by microwave plasma CVD with nitrogen addition. Diamond and Related Materials, 15, pp. 455-459.

Nasdala, L., D.C. Smith, R. Kaindl, M.A. Zieman 2004: Spectroscopic methods in mineralogy. - In: Beran, A., E. Libowitzky (Eds.) Raman spectroscopy: Analytical perspectives in mineralogical research. EMU Notes In Mineralogy 6, Eötvös University Press, pp. 281-343. 
Nuth, J.A.III., J.E. Allen Jr. 1992: Supernovae as sources of interstellar diamonds. - Astrophysics and Space Sciences, 196, pp. 117-123.

Ozima, M., M. Tatsumoto 1997: Radiation-induced diamond crystallization: origin of carbonados and its implications on meteorite nano-diamonds. - Geochimica et Cosmochimica Acta, 61, pp. 369-376.

Richter, S., U. Ott, F. Begemann 1998: Tellurium in presolar diamonds as an indicator for rapid separation of supernova ejecta. - Nature, 391, p. 261.

Russel, S.S., J.W. Arden, C.T. Pillinger 1992: A new type of meteoritic diamond in the enstatite chondrite Abee. - Science, 256, no. 5054 pp. 206-209.

Sun, L., J. Gong, D. Zhu, Z. Zhu, S. He 2004: Diamond nanorods from carbon nanotubes. Advanced Materials, 16, pp. 1849-1853.

Sun, Z., J.R. Shi, B.K. Tay, S.P. Lau 2000: UV raman characteristics of nanocrystalline diamond films with different grain size. - Diamond Related Materials, 9, pp. 1979-1983.

Tielens, A.G.G.M., C.G. Sseab, D.J. Hollenbach, C.F. McKee 1987: Shock processing of interstellar dust - Diamonds in the sky. - The Astrophysical Journal, 319, L109-L113.

Zhang, D., R.Q. Zhang. 2005: Convergence in the evolution of nanodiamond Raman spectra with particle size: A theoretical investigation. - Journal of Physics and Chemistry B., 109, pp. 9006-9013.

Zinner, E. 1998: Stellar nucleosynthesis and the isotopic composition of presolar grains from primitive meteorites. - Annual Review of Earth and Planetary Sciences, 26, pp. 147-188.

Yoshikawa, M., Y. Mori, H. Obata, M. Maegawa, G. Katagiri, H. Ishida, A. Ishitani 1995: Raman scattering from nanometer-sized diamond. - Applied Physics Letters, 67, 1995, pp. 694-697. 